\title{
A Case of Psychogenic Belching
}

\author{
Sharma $\mathbf{P}^{1}$, Sharma PP2 , Chaudhary DP³ Bastola $\mathbf{P}^{1}$
}

\begin{abstract}
1. Medical Officer, Mental Disease Treatment and Rehabilitation Centre, Jorpati Attarkhel, Kathmandu 2 Professor, Department of Psychiatry, Kathmandu University School of Medicine 3. Medical Officer, Norvic International Hospital, Thapathali, Kathmandu
\end{abstract}

E-mail *Corresponding author: drpushpasharma@yahoo.com

\section{Abstract}

Belching is the act of expelling air from the stomach through the mouth. It usually occurs when the stomach distends (expands) due to too much swallowed air, with the belching releasing the air to reduce the distention. We report here a case of psychogenic belching who was referred to psychiatry department after 3 months of her symptoms who responded to treatment quite effectively.

Key words: Psychogenic belching, Conversion Disorder, Nepal

\section{INTRODUCTION}

Belching is the act of expelling air from the stomach through the mouth. It usually occurs when the stomach distends (expands) due to too much swallowed air, with the belching releasing the air to reduce the distention.

Belching may be a primary presenting complaint that is socially debilitating and difficult to treat. Attention has been found to modify belching frequency such that some cases may be psychogenic. Leibovich et. al.1 defined psychogenic nausea and vomiting as vomiting without any obvious organic pathology or with a psychological etiology. This case was reported to describe a case of Conversion Disorder presented as persistent continuous belching and to discuss psychological aspect of the problem.

\section{CASE REPORT}

14 year old girl studying in grade 9 had developed belching unabated since 3 months. The onset of belching was acute with no nausea and vomiting. According to the family member, her belching would continue throughout the day but would stop while sleeping. She was eventually addressed by Gastroenterologist and was diagnoses as having Acute Gastritis. Upper G.I endoscopy was done which showed mild antral gastritis.
She was treated with Proton Pump Inhibitors along with 5HT3 blocker for her Acute Gastritis problem. However, after 2 weeks of treatment she did not yield any significant improvement in her symptoms.

Although her belching continued significantly even after the treatment, there was no weight loss and her appetite was fairly normal. There was no dysphagia.

She was one of the top students of her class. But due to the socially distressing nature of the symptoms she was asked not to attend school by her parents.

Ultimately She was referred to the Psychiatry Unit after 3 months of her persistent symptoms.

In an interview with her caretaker (Grand mother), she revealed that her granddaughter was stubborn, demanding and would react angrily to any criticism and to her unmet demands.

They had a joint family where her Grandparents, her working parents and her 5 year younger sister lived, with whom she had a visible sibling rivalry.

There was discrepancy in parenting modalities between her parents and her grandparents.

On Examination: She was well kept, tidy and cooperative.

Her speech was normal. Higher Mental Function was intact. There was no evidence of 
depressive illness, psychotic features or other organic mental disorder.

She had mild irritability and showed no distress over her belching despite having these distressful symptoms for a long time. She did not show any concern about her disruption in her academics despite she being a good student.

She was managed with Clonazepam $0.25 \mathrm{mg}$ twice a day and escitalopram $5 \mathrm{mg}$ once a day. She was conducted about 14 sessions of intensive behavior therapy. By the end of 10 sessions, she started showing improvement in her belching frequencies and by the end of 12 sessions her belching completely stopped.

Simultaneously, her counseling sessions were conducted along with her parents where they were given the acumen of parenteral handling in this case. All faulty parenteral handling employed by her parents were explained and was advised to rectify.

\section{DISCUSSION}

This case belongs to conversion disorder, where primary symptoms of belching presents as a change in physical functioning. We could see from the history and also from the physical examination that, the patient has reacted to the illness with an attitude of indifference and showing amazing lack of concern.

There are evidences to support in this case that, she has a strong histrionic personality trait and that may be one of the contributory factor in the genesis of this disorder. The tangible secondary gain in this case being the excessive attention she gets from both the working parents. Also in our history there was significant sibling rivalry between her and her younger sister who was younger to her by 5 years.

This case reveals the necessity of medical professional of different sub specialty in medical sciences needs to be well versed on the possibility of patients presenting with physical symptoms with underlying psychiatric illnesses, for early diagnosis and timely management. It will help save time, resources and reduce family distress hence decreasing the overall burden of care.
In a randomized trial ${ }^{2}, 26$ functional dyspepsia patients were randomized to hypnotherapy, supportive psychotherapy plus placebo medication, or medical treatment for 16 weeks. ${ }^{1}$ Compared with the other approaches, hypnotherapy significantly improved symptoms in short term or long-term treatment. ${ }^{3}$

\section{REFERENCE}

1. Leibovich MA. Psychogenic vomiting. Psychotherapeutic considerations. Psychother Psychosom 1973;22:263-8

2. Cheng C, Yang FC, Jun S, et al. Flexible coping psychotherapy for functional dyspeptic patients: a randomized, controlled trial. Psychosom Med 2007;69:81-8.

3. Calvert EL, Houghton LA, Cooper P, et al. Long-term improvement in Functional dyspepsia using Hypnotherapy. Gastroenterology 2002;123:1778-85. prevention, 1994; 26:325-37. 